\title{
Antenna selection in MIMO radar with collocated antennas
}

\author{
ZHANG Haowei ${ }^{1,{ }^{*}}$, XIE Junwei ${ }^{1}$, SHI Junpeng ${ }^{2}$, and ZHANG Zhaojian ${ }^{3}$ \\ 1. Air and Missile Defense College, Air Force Engineering University, Xi' an 710051, China; \\ 2. Electromagnetic Countermeasure College, National University of Defense Technology, Hefei 230031, China; \\ 3. Air Force Early Warning Academy of the PLA, Wuhan 410039, China
}

\begin{abstract}
An antenna adjustment strategy is developed for the target tracking problem in the collocated multiple-input multipleoutput (MIMO) radar. The basic technique of this strategy is to optimally allocate antennas by the prior information in the tracking recursive period, with the objective of enhancing the worst-case estimate precision of multiple targets. On account of the posterior Cramer-Rao lower bound (PCRLB) offering a quantitative measure for target tracking accuracy, the PCRLB of joint direction-of-arrival (DOA) and Doppler is derived and utilized as the optimization criterion. It is shown that the dynamic antenna selection problem is NP-hard, and an efficient technique which combines convex relaxation with local search is put forward as the solution. Simulation results demonstrate the outperformance of the proposed strategy to the fixed antenna configuration and heuristic search algorithm. Moreover, it is able to offer close-to performance of the exhaustive search method.
\end{abstract}

Keywords: collocated multiple-input multiple-output (MIMO) radar, multi-target tracking, antenna selection, posterior CramerRao lower bound (PCRLB), direction-of-arrival (DOA).

DOI: $10.21629 /$ JSEE.2019.06.08

\section{Introduction}

\subsection{Background and motivation}

The collocated multiple-input multiple-output (MIMO) radar has attracted much attention in the recent years [14]. Such a system shows significant advantages, such as virtual aperture extension, path diversity [1], and higher probability of detection [2], over traditional phased array radars. The research scope varies from target detection $[5,6]$, target localization [7-10], and target tracking $[11,12]$. However, in practice, the resources in the radar system are usually limited. Consequently, it generates much interest among researchers to investigate the resource-aware design in the collocated MIMO radar.

\footnotetext{
Manuscript received January 03, 2019.

*Corresponding author.

This work was supported by the National Natural Science Foundation of China (61601504).
}

In [7-12], the Cramer-Rao lower bound (CRLB), which provides a tight lower bound for any unbiased estimator, is derived as an evaluation tool. In [7], the CRLB of the direction-of-arrival (DOA) estimate was firstly studied, and the object is the single static target. However, when multiple targets fall into the same resolution cell, the CRLB will be affected accordingly. Thus, Li et al. [8] proposed an alternative form of CRLB, where multiple targets occupying the same resolution cell is considered. Corji et al. $[9,10]$ further analyzed the influence of the time delay, and put forward the CRLB of joint range and DOA. The CRLB associated with the DOA estimate was also investigated in the bistatic MIMO radar in [13]. However, the study in [7-10] and [13] is based on the single pulse, while the situation of pulse train is not discussed. For target tracking, Yan et al. [11,12] derived the posterior CRLB (PCRLB) [14] of range, velocity and angle, and the trace of PCRLB is chosen as the criterion for the power allocation. However, the precondition of multiple targets being well separated and one beam tracking one target should be highlighted [15]. When there are overlaps among multiple beams, which is more often faced in applications, a new system model should be explored.

Antenna configuration plays an important role in the resource-aware design of sensor systems. Bishop et al. [16] respectively identified the optimal relative sensor-target geometries by using the CRLB of target localization on bearing-only, range-only, and time-of-arrival-based as the criterion. In the scenario of target tracking, Tharmarasa et al. $[17,18]$ utilized the PCRLB to find optimal subsets of large-scale sensors, where the number of targets in the surveillance region being fixed and known as well as time varying and unknown are separately studied. The CRLB was also employed in [19-23] to guideline the antenna allocation in the widely separated MIMO radar. By dynamically choosing the subsets of antennas, the target state estimate accuracy is evidently enhanced. However, the work on the antenna allocation in collocated MIMO radar is far 
limited. When such a system is operated over a long period, the cost of operation becomes significant [20]. Particularly, in the hostile environment, to meet the requirement of low-probability of interception, the number of antennas to be activated is finite. Furthermore, it has been shown in $[9,10]$ that, in collocated MIMO radar, the CRLB of localization is the function of antenna placement. Therefore, it is an interesting topic of optimally selecting the antennas in a novel scenario of target tracking. While Gorji et al. [9] has investigated the antenna placement for the collocated MIMO radar, the following points should be emphasized.

(i) The scenario is for the static target localization, but not applicable for tracking moving targets. The time delay and DOA are the focus in the former case, while the Doppler shift and DOA are the concern in the latter case.

(ii) An antenna placement model was established in [9]. However, in the application, the antenna locations cannot be timely tuned in a designed collocated MIMO radar. Therefore, the optimization model of antenna selection is more practical.

(iii) As mentioned before, reference [9] pays attention to the receiving signal containing the single pulse, but the situation of the pulse train is neglected.

\subsection{Methodology}

For the target tracking, the cognition technique [24] establishes a feedback framework from the transmitter to the receiver, so that perceives the change of environment and then adapts the working parameters. Therefore, it is exploited in various resource management issues for the MIMO radar [11,12,25-27]. In the collocated MIMO radar, Yan et al. $[11,12]$ put forward an efficient power allocation strategy in the simultaneous multi-beam working mode by exploiting the target prior information. The waveform adaption was further considered in [25]. In the distributed MIMO radar, Chavali et al. [26] proposed a greedy search based algorithm for the joint sensor and power allocation according to the target state and channel state. Garcia et al. [27] investigated the joint power and bandwidth allocation for multi-target localization.

\subsection{Main contributions}

As such, this paper concerns the antenna allocation problem in the multi-target tracking by collocated MIMO radar, where the joint DOA and Doppler shift estimate model is established in the situation of transmitting coherent pulse train, and a dynamic antenna selection scheme is proposed. The main contributions are as follows:

(i) The PCRLB of Doppler shift and DOA is derived with transmitting coherent pulse train. In comparison with the case of single pulse, the accumulation of multiple pulses should be considered in such condition. Therefore, two representative situations of target radar cross section (RCS), i.e., Swerling I model and Swerling II model, which affect the accumulation, are discussed. To describe the overall tracking precison, the optimization model is formulated by regarding the finite active antennas to achieve the minimum of PCRLB in the worst case among multiple targets.

(ii) An efficient method is put forward for the solution to both cases. In the combinatorial optimization framework, the subset selection issue is NP-hard [28]. While the exhaustive search [28] or heuristic search [19,20,22] is transferable, either the computationally complex or only localoptima in performance is beyond satisfactory. To solve this problem in an efficient manner, we first relax the original model and reformulate it as a convex one [15,29]. Then, a local search around the solution to the convex model is adopted for a higher precision.

(iii) A closed loop feedback scheme for antenna configuration is established. The square-root cubature Kalman filter (SCKF) [30] is used to tackle the nonlinear transform and to acquire an accurate target state estimate. After getting the tracking information from current time epoch, the PCRLB is predicted and used as the guideline of antenna adjustment for the next time epoch. Thereby, the MIMO radar can adaptively tune the antenna configuration to respond to the change of the target state, and a closed loop tracking system is developed.

(iv) The simulation results demonstrate the outperformance of the proposed strategy than the fixed antenna configuration and heuristic search algorithm [20]. Moreover, it provides close-to optimal performance while being enough efficient.

The rest of this article is as follows. Section 2 depicts the system models. In Section 3, the predicted PCRLB related to antenna adjustment is derived. In Section 4, the optimization problem is established and an efficient algorithm is put forward for the solution. Section 5 presents the simulation results and analysis. The conclusions of this paper is given in Section 6.

\section{System model}

We first establish the target tracking model in the collocated MIMO radar, which can provide a basis for the derivation of predicted PCRLB.

\subsection{Signal model}

Consider a narrowband collocated MIMO radar, whose center is located at the original point $(0,0)$. The location of the $m$ th transmit array is denoted as $\left(x_{t m}, y_{t m}\right)$, and the $n$th receive array is denoted as $\left(x_{r n}, y_{r n}\right)$, where 
$m=1,2, \ldots, M$ and $n=1,2, \ldots, N$. Each array transmits orthogonal and coherent pulse train signals. The pulse width is $t_{c}$, and it is small enough so that the target RCS remains to be constant in once pulse duration. The signal transmitted by the $m$ th transmit array is denoted as $s_{m}(t)$, the pulse repetition period is denoted as $T_{p}$, and the carrier wavelength is denoted as $\lambda$. The number of transmit pulse train in once measurement is denoted as $L$. Therefore, in the $k$ th sample interval, the $l$ th pulse in $s_{m}(t)$ is

$$
s_{k, m, l}(t)=s_{k, m}\left(t^{\prime}+(l-1) T_{p}\right), \quad 0 \leqslant t^{\prime} \leqslant t_{c}
$$

where $t$ and $t^{\prime}$ are the fast time and the slow time, respectively. There are $Q$ targets moving on the $x-y$ plane. By the time delay compensation, the $l$ th pulse in the received signal from the $q$ th target is expressed $[9,13]$ as

$$
\boldsymbol{s}_{r k, l}^{q}(t)=\alpha_{k, l}^{q} \boldsymbol{a}_{r}\left(\theta_{k}^{q}\right) \boldsymbol{a}_{t}^{\mathrm{T}}\left(\theta_{k}^{q}\right) \boldsymbol{s}_{k, l}(t) \mathrm{e}^{-\mathrm{j} 2 \pi f_{d k}^{q} t}+\boldsymbol{n}_{k, l}(t)
$$

$$
\left\{\begin{array}{l}
{\left[\boldsymbol{a}_{r}\left(\theta_{k}^{q}\right)\right]_{n}=\exp \left[-\mathrm{j} \frac{2 \pi}{\lambda}\left(\cos \left(\theta_{k}^{q}\right) x_{r n}+\sin \left(\theta_{k}^{q}\right) y_{r n}\right)\right]} \\
{\left[\boldsymbol{a}_{t}\left(\theta_{k}^{q}\right)\right]_{m}=\exp \left[-\mathrm{j} \frac{2 \pi}{\lambda}\left(\cos \left(\theta_{k}^{q}\right) x_{t m}+\sin \left(\theta_{k}^{q}\right) y_{t m}\right)\right]} \\
{\left[\boldsymbol{s}_{k, l}(t)\right]_{m}=s_{k, m, l}(t)}
\end{array}\right.
$$

where $\alpha_{k, l}^{q}$ is the target RCS. $f_{d k}^{q}$ is the Doppler frequency shift. $[\cdot]_{i}$ denotes the $i$ th element in the vector. $\boldsymbol{a}_{r}\left(\theta_{k}^{q}\right)$ and $\boldsymbol{a}_{t}\left(\theta_{k}^{q}\right)$ are the receive steering vector and the transmit steering vector respectively. $\theta_{k}^{q}$ is the target angle. $\boldsymbol{s}_{k, l}(t)$ is the transmit signal vector. $\boldsymbol{a}_{r}\left(\theta_{k}\right) \in \mathbb{C}^{N \times 1}$, $\boldsymbol{a}_{t}\left(\theta_{k}\right) \in \mathbb{C}^{M \times 1}$ and $\boldsymbol{s}_{k, l}(t) \in \mathbb{C}^{M \times 1} \cdot \boldsymbol{n}_{k, l}(t)$ is the zero mean Gaussian noise.

Substituting (2) into the slow time, and via the coherent process [9], we have

$$
\begin{gathered}
\boldsymbol{r}_{k, l}^{q}=\alpha_{k, l}^{q} \mathrm{e}^{-\mathrm{j} 2 \pi f_{d k}(l-1) T_{p}} \operatorname{vec}\left(\boldsymbol{a}_{r}\left(\theta_{k}^{q}\right) \boldsymbol{a}_{t}^{\mathrm{T}}\left(\theta_{k}^{q}\right)\right)+\boldsymbol{v}_{k, l}= \\
\alpha_{k, l}^{q} \mathrm{e}^{-\mathrm{j} 2 \pi f_{d k}(l-1) T_{P}} \boldsymbol{g}_{k}^{q}+\boldsymbol{v}_{k, l}
\end{gathered}
$$

where $\operatorname{vec}(\cdot)$ denotes the vectorizing operation to the matrix. $\boldsymbol{v}_{k, l} \sim \mathcal{N}\left(0, \sigma_{v}^{2} \boldsymbol{I}_{M N}\right)$, i.e., the complex Gaussian vector with zero mean and covariance of $\sigma_{v}^{2} \boldsymbol{I}_{M N} . \boldsymbol{I}_{M N}$ is the $M N \times M N$ identity matrix. $\boldsymbol{g}_{k}^{q}$ is expressed as

$$
\begin{gathered}
\boldsymbol{g}_{k}^{q}=\exp \left[j \frac { j \pi } { \lambda } \left(\boldsymbol{B}_{t} \otimes \mathbf{1}_{1 \times N}-\right.\right. \\
\left.\left.\mathbf{1}_{1 \times M} \otimes \boldsymbol{B}_{r}\right)^{\mathrm{T}}\left[\cos \theta_{k}^{q}, \sin \theta_{k}^{q}\right]^{\mathrm{T}}\right] \\
\begin{cases}\boldsymbol{B}_{t}=\left[\boldsymbol{b}_{t 1}, \boldsymbol{b}_{t 2}, \ldots, \boldsymbol{b}_{t M}\right], & \boldsymbol{b}_{t m}=\left[x_{t m}, y_{c m}\right]^{\mathrm{T}} \\
\boldsymbol{B}_{r}=\left[\boldsymbol{b}_{r 1}, \boldsymbol{b}_{r 2}, \ldots, \boldsymbol{b}_{r N}\right], & \boldsymbol{b}_{r n}=\left[x_{r n}, y_{r n}\right]^{\mathrm{T}}\end{cases}
\end{gathered}
$$

where $\otimes$ denotes the Kronecker product. $\mathbf{1}_{N \times 1}$ denotes the $N \times 1$ vector with all entries being equal to one. $\boldsymbol{b}_{t m}$ is the location of the $m$ th transmit array, and $\boldsymbol{b}_{r n}$ is the location of the $n$th receive array.

\subsection{Target dynamics}

Assume that the target motion model can be described by the constant acceleration (CA) model [31]:

$$
\boldsymbol{x}_{k+1}^{q}=\boldsymbol{F} \boldsymbol{x}_{k}^{q}+\boldsymbol{w}_{k}^{q}
$$

where $\boldsymbol{x}_{k}^{q}=\left[x_{k}^{q}, \dot{x}_{k}^{q}, \ddot{x}_{k}^{q}, y_{k}^{q}, \dot{y}_{k}^{q}, \ddot{y}_{k}^{q}\right]^{\mathrm{T}} . \boldsymbol{F}$ is the transition matrix with

$$
\boldsymbol{F}=\boldsymbol{I}_{2} \otimes\left[\begin{array}{ccc}
1 & T_{s} & T_{s}^{2} / 2 \\
0 & 1 & T_{s} \\
0 & 0 & 1
\end{array}\right]
$$

where $T_{s}$ is the sample interval, and the process noise $\boldsymbol{w}_{k}^{q} \sim \mathcal{N}\left(\mathbf{0}, \boldsymbol{Q}_{q}\right)$. In addition, we have (9) with the covariance of the process noise:

$$
\boldsymbol{Q}_{q}=\kappa_{q} \boldsymbol{I}_{2} \otimes\left[\begin{array}{ccc}
T_{s}^{5} / 20 & T_{s}^{4} / 8 & T_{s}^{3} / 6 \\
T_{s}^{4} / 8 & T_{s}^{3} / 3 & T_{s}^{2} / 2 \\
T_{s}^{3} / 6 & T_{s}^{2} / 2 & T_{s}
\end{array}\right]
$$

where $\kappa_{q}$ is the process noise intensity [15].

\subsection{Measurement model}

From the receive signal described in (4), some target information can be extracted:

$$
\boldsymbol{z}_{k}^{q}=h\left(\boldsymbol{x}_{k}^{q}\right)+\boldsymbol{\eta}_{k}^{q} .
$$

Here, we focus on the Doppler shift and angle. As such, $h(\cdot)$ is the nonlinear transform with respect to

$$
\left\{\begin{array}{l}
\dot{r}_{k}^{q}=\left(x_{k}^{q} \dot{x}_{k}^{q}+y_{k}^{q} \dot{y}_{k}^{q}\right) / \sqrt{\left(x_{k}^{q}\right)^{2}+\left(y_{k}^{q}\right)^{2}} \\
\theta_{k}^{q}=\arctan \left(y_{k}^{q} / x_{k}^{q}\right)
\end{array} .\right.
$$

Without loss of generality, we assume that the covariance matrix of $\boldsymbol{\eta}_{k}^{q}$ is $\boldsymbol{R}_{k}^{q}$, and $\boldsymbol{R}_{k}^{q}$ is given by the CRLB matrix, which will be shown in the next section.

\section{PCRLB of joint velocity and DOA}

Before the antenna adaption, an optimization criterion should be taken into account. Especially in target tracking, such as a recursive cycle, the criterion should be predictive and not be influenced by the adopted filtering algorithm. Therefore, the PCRLB is chosen to perform this function.

For the target measurement model depicted in Section 2, (12) are obtainable when we assume that $f\left(\boldsymbol{r}_{k}^{q}\right)$ is the unbiased estimate [14] for $\boldsymbol{x}_{k}^{q}$.

$$
\mathbb{E}_{\boldsymbol{x}_{k}^{q}, \boldsymbol{r}_{k}^{q}}\left[\left(f\left(\boldsymbol{r}_{k}^{q}\right)-\boldsymbol{x}_{k}^{q}\right)\left(f\left(\boldsymbol{r}_{k}^{q}\right)-\boldsymbol{x}_{k}^{q}\right)^{\mathrm{T}}\right] \geqslant \boldsymbol{J}^{-1}\left(\boldsymbol{x}_{k}^{q}\right)
$$

where $\mathbb{E}_{\boldsymbol{x}_{k}^{q}, \boldsymbol{r}_{k}^{q}}$ denotes the expectation operator. $\boldsymbol{J}\left(\boldsymbol{x}_{k}^{q}\right)$ is the Bayesian fisher information matrix (BIM) about the 
target state. For simplicity, we just give the final expression of $\boldsymbol{J}\left(\boldsymbol{x}_{k}^{q}\right)$, and more details can be referred to [11] and [14]:

$$
\boldsymbol{J}\left(\boldsymbol{x}_{k}^{q}\right)=\boldsymbol{J}_{p}\left(\boldsymbol{x}_{k}^{q}\right)+\boldsymbol{J}_{D}\left(\boldsymbol{x}_{k}^{q}\right)
$$

where $\boldsymbol{J}_{P}\left(\boldsymbol{x}_{k}^{q}\right)$ and $\boldsymbol{J}_{D}\left(\boldsymbol{x}_{k}^{q}\right)$ are the Fisher information matrices of the prior information and the data, respectively $[12,15]$ :

$$
\begin{gathered}
\boldsymbol{J}_{P}\left(\boldsymbol{x}_{k}^{q}\right)=\left[\boldsymbol{Q}_{q}+\boldsymbol{F} \boldsymbol{J}^{-1}\left(\boldsymbol{x}_{k-1}^{q}\right) \boldsymbol{F}^{\mathrm{T}}\right]^{-1} \\
\boldsymbol{J}_{D}\left(\boldsymbol{x}_{k}^{q}\right)=\mathbb{E}_{\boldsymbol{x}_{i}}\left[\left(\boldsymbol{H}_{k}^{q}\right)^{\mathrm{T}}\left(\boldsymbol{R}_{k}^{q}\right)^{-1} \boldsymbol{H}_{k}^{q}\right]
\end{gathered}
$$

where $\boldsymbol{H}_{k}^{q}$ is the Jacobian matrix of the measurement function $h(\cdot)$ with respect to the target state $\boldsymbol{x}_{k}^{q}$. The closed form $\boldsymbol{R}_{k}^{-1}$ is derived as follows, where the target RCS complying with Swering I model and Swerling II model are both discussed.

We note that throughout the derivation, the target index $q$ is always omitted unless doing so will cause confusion.

On the condition of Swerling I and Swerling II models, the target's reflectivity $\alpha$ follows

$$
\alpha=\sqrt{\Sigma} \mathrm{e}^{\mathrm{j} \Omega}
$$

where $\Omega$ follows the even distribution, and the probability density function (PDF) of $\Sigma$ is

$$
p(\Sigma)=\frac{1}{\bar{\sigma}} \mathrm{e}^{-\Sigma / \bar{\sigma}}, \quad \Sigma \geqslant 0
$$

where $\bar{\sigma}$ is the mean reflectivity. In Swerling I model, $\alpha$ remains constant during one pulse train. By contrast, in Swerling II model, $\alpha$ changes from pulse to pulse, and each $\alpha$ is individual.

On the condition of Swerling I model, we have

$$
\alpha_{k, l}=\alpha_{k} .
$$

Combining (4), (10) and (18), the measurement matrix in time epoch $k$ can be rewritten as

$$
\boldsymbol{r}_{k}=\alpha_{k} \boldsymbol{G}_{k} \boldsymbol{c}_{\mathrm{I} k}+\boldsymbol{v}_{k}
$$

where

$$
\begin{gathered}
\boldsymbol{G}_{k}=\operatorname{diag}\left\{\boldsymbol{g}_{k}, \boldsymbol{g}_{k}, \ldots, \boldsymbol{g}_{k}\right\}_{L} \\
\boldsymbol{c}_{\mathrm{I} k}=\left[1, \mathrm{e}^{-\mathrm{j} 2 \pi f_{d k} T_{p}}, \ldots, \mathrm{e}^{-\mathrm{j} 2 \pi f_{d k}(L-1) T_{p}}\right]^{\mathrm{T}}
\end{gathered}
$$

where $\operatorname{diag}\{\cdot\}_{L}$ denotes that there are $L$ diagonal elements in the matrix.

From (19), we have

$$
\begin{gathered}
p\left(\boldsymbol{r}_{k} \mid \boldsymbol{\xi}_{k}\right)= \\
\frac{1}{\pi^{M N L} \sigma_{\boldsymbol{v}}^{2 M N L}} \exp \left[-\frac{\left\|\boldsymbol{r}_{k}-\alpha_{k} \boldsymbol{G}_{k} \boldsymbol{c}_{\mathrm{I}}\right\|^{2}}{\sigma_{\boldsymbol{v}}^{2}}\right]
\end{gathered}
$$

where $\boldsymbol{\xi}_{k}=\left[\dot{r}_{k}, \theta_{k}\right]^{\mathrm{T}}$. We introduce an intermediate variable $\boldsymbol{\mu}_{\mathrm{I} k}=\alpha_{k} \boldsymbol{G}_{k} \boldsymbol{c}_{\mathrm{I} k}$, then, the $[p q]$ th element in $\boldsymbol{R}_{k}^{-1}$ is

$$
\left(\boldsymbol{R}_{\mathrm{I} k}^{-1}\right)_{p q}=\frac{2}{\sigma_{\boldsymbol{v}}^{2}} \operatorname{Re}\left(\frac{\partial \boldsymbol{\mu}_{\mathrm{I} k}^{\mathrm{H}}}{\partial\left(\boldsymbol{\xi}_{k}\right)_{p}} \frac{\partial \boldsymbol{\mu}_{\mathrm{I} k}}{\partial\left(\boldsymbol{\xi}_{k}^{\mathrm{T}}\right)_{q}}\right) .
$$

When $p=2$ and $q=2$, we have

$$
\begin{gathered}
\left(\boldsymbol{R}_{\mathrm{I} k}^{-1}\right)_{22}=\frac{2}{\sigma_{\boldsymbol{v}}^{2}} \operatorname{Re}\left(\frac{\partial \boldsymbol{\mu}_{\mathrm{I} k}^{\mathrm{H}}}{\partial \theta_{k}} \frac{\partial \boldsymbol{\mu}_{\mathrm{I} k}}{\partial \theta_{k}}\right)= \\
\frac{2\left|\alpha_{k}\right|^{2}}{\sigma_{\boldsymbol{v}}^{2}} \operatorname{Re}\left(\boldsymbol{c}_{\mathrm{I} k}^{\mathrm{H}} \frac{\partial \boldsymbol{G}_{k}^{\mathrm{H}}}{\partial \theta_{k}} \frac{\partial \boldsymbol{G}_{k}}{\partial \theta_{k}} \boldsymbol{c}_{\mathrm{I} k}\right) .
\end{gathered}
$$

The calculation on $\partial \boldsymbol{G}_{k}^{\mathrm{H}} / \partial \theta_{k}$ is equivalent to $\partial \boldsymbol{g}_{k}^{\mathrm{H}} / \partial \theta_{k}$, and the latter item is easily obtained as

$$
\begin{gathered}
\frac{\partial \boldsymbol{g}_{k}^{\mathrm{H}}}{\partial \theta_{k}} \frac{\partial \boldsymbol{g}_{k}}{\partial \theta_{k}}=\left(\frac{2 \pi}{\lambda}\right)^{2}\left(\sum_{m=1}^{M} \sum_{n=1}^{N}\left[\sin \theta_{k},-\cos \theta_{k}\right] .\right. \\
\left.\Delta \boldsymbol{b}_{m n}\left(\Delta \boldsymbol{b}_{m n}\right)^{\mathrm{T}}\left[\sin \theta_{k},-\cos \theta_{k}\right]^{\mathrm{T}}\right)
\end{gathered}
$$

where

$$
\Delta \boldsymbol{b}_{m n}=\boldsymbol{b}_{t m}-\boldsymbol{b}_{r n} .
$$

Therefore, (24) is rewritten as

$$
\begin{gathered}
\left(\boldsymbol{R}_{\mathrm{I} k}^{-1}\right)_{22}=\frac{2\left|\alpha_{k}\right|^{2} L}{\sigma_{\boldsymbol{v}}^{2}}\left(\frac{2 \pi}{\lambda}\right)^{2}\left(\sum_{m=1}^{M} \sum_{n=1}^{N}\left[\sin \theta_{k},-\cos \theta_{k}\right]\right. \\
\left.\Delta \boldsymbol{b}_{m n}\left(\Delta \boldsymbol{b}_{m n}\right)^{\mathrm{T}}\left[\sin \theta_{k},-\cos \theta_{k}\right]^{\mathrm{T}}\right)
\end{gathered}
$$

Meanwhile, we have

$$
\left(\boldsymbol{R}_{\mathrm{I} k}^{-1}\right)_{11}=\frac{32\left|\alpha_{k}\right|^{2} \pi^{2} T_{p}^{2} M N}{\sigma_{\boldsymbol{v}}^{2} \lambda^{2}} \frac{(L-1) L(2 L-1)}{6} .
$$

In Swering II model, we have (29) by combining (4) and (10)

$$
\boldsymbol{r}_{k}=\boldsymbol{G}_{k} \boldsymbol{C}_{\mathrm{II} k} \boldsymbol{\alpha}_{k}+\boldsymbol{v}_{k}
$$

where

$$
\begin{gathered}
\boldsymbol{C}_{\mathrm{II} k}=\operatorname{diag}\left\{1, \mathrm{e}^{-\mathrm{j} 2 \pi f_{d k} T_{p}}, \ldots, \mathrm{e}^{-\mathrm{j} 2 \pi f_{d k}(L-1) T_{p}}\right\} \\
\boldsymbol{\alpha}_{k}=\left[\alpha_{1}, \alpha_{2}, \ldots, \alpha_{L}\right]^{\mathrm{T}} .
\end{gathered}
$$

When regarding $\alpha$ as a determining variable,

$$
\begin{gathered}
p\left(\boldsymbol{r}_{k} \mid \boldsymbol{\xi}_{k}\right)= \\
\frac{1}{\pi^{M N L} \sigma_{\boldsymbol{v}}^{2 M N L}} \exp \left[-\frac{\left\|\boldsymbol{r}_{k}-\boldsymbol{G}_{k} \boldsymbol{C}_{\mathrm{II} k} \boldsymbol{\alpha}_{k}\right\|^{2}}{\sigma_{\boldsymbol{v}}^{2}}\right] .
\end{gathered}
$$

Then, the $[p q]$ th element in $\boldsymbol{R}_{k}^{-1}$ is as follows after introducing $\boldsymbol{\mu}_{\mathrm{II} k}=\boldsymbol{G}_{k} \boldsymbol{C}_{\mathrm{II} k} \boldsymbol{\alpha}_{k}$ :

$$
\left(\boldsymbol{R}_{\mathrm{II} k}^{-1}\right)_{p q}=\frac{2}{\sigma_{\boldsymbol{v}}^{2}} \operatorname{Re}\left(\frac{\partial \boldsymbol{\mu}_{\mathrm{II} k}^{\mathrm{H}}}{\partial\left(\boldsymbol{\xi}_{k}\right)_{p}} \frac{\partial \boldsymbol{\mu}_{\mathrm{II} k}}{\partial\left(\boldsymbol{\xi}_{k}^{\mathrm{T}}\right)_{q}}\right) .
$$


When $p=2$ and $q=2$, we have

$$
\begin{gathered}
\left(\boldsymbol{R}_{\mathrm{II} k}^{-1}\right)_{22}=\frac{2}{\sigma_{\boldsymbol{v}}^{2}}\left(\sum_{l=1}^{L} \alpha_{l}^{*} \alpha_{l}\right)\left(\frac{2 \pi}{\lambda}\right)^{2} . \\
\left(\sum_{m=1}^{M} \sum_{n=1}^{N}\left[\cos \theta_{k},-\sin \theta_{k}\right] \cdot\right. \\
\left.\Delta \boldsymbol{b}_{m n}\left(\Delta \boldsymbol{b}_{m n}\right)^{\mathrm{T}}\left[\cos \theta_{k},-\sin \theta_{k}\right]^{\mathrm{T}}\right) .
\end{gathered}
$$

Similar with (27), the analytic expression of (34) shows that the antenna configuration influences the estimation performance.

Additionally, we have

$$
\left(\boldsymbol{R}_{\mathrm{II} k}^{-1}\right)_{11}=\frac{32 M N \pi^{2} T_{p}^{2}}{\sigma_{\boldsymbol{v}}^{2} \lambda^{2}}\left(\sum_{l=1}^{L} \alpha_{l}^{*} \alpha_{l}(l-1)^{2}\right) .
$$

Finally, we will have the BIM expression:

$$
\begin{aligned}
& \boldsymbol{J}\left(\boldsymbol{x}_{k}^{q}\right)=\left(\boldsymbol{Q}_{q}+\boldsymbol{F} \boldsymbol{J}^{-1}\left(\boldsymbol{x}_{k-1}^{q}\right) \boldsymbol{F}^{\mathrm{T}}\right)^{-1}+ \\
& \mathbb{E}_{\boldsymbol{x}_{k}^{q}}\left[\left(\boldsymbol{H}_{k}^{q}\right)^{\mathrm{T}}\left(\boldsymbol{R}_{k}^{q}\right)^{-1} \boldsymbol{H}_{k}^{q}\right] .
\end{aligned}
$$

From the derivation above, we can see that the transmit and receive antenna configurations have an impact on $\left(\boldsymbol{R}_{k}^{q}\right)^{-1}$, and thus on $\boldsymbol{J}\left(\boldsymbol{x}_{k}^{q}\right)$. It naturally leads to optimizing $\boldsymbol{J}\left(\boldsymbol{x}_{k}^{q}\right)$ by the change of antenna selection dynamically. However, the main problem in the application of (36) is that, the expectation operator in the BIM will be evaluated by the Monte Carlo techniques [12]. That means, to achieve the CRLB, we must implement a large number of Monte Carlo simulations, which will consume too much time. To satisfy the real-time demand of radar system, we use $\widehat{\boldsymbol{H}}_{k}^{q}$ and $\widehat{\boldsymbol{R}}_{k}^{q}$ to approximate $\boldsymbol{H}_{k}^{q}$ and $\boldsymbol{R}_{k}^{q}$ respectively, where $\widehat{\boldsymbol{H}}_{k}^{q}$ and $\widehat{\boldsymbol{R}}_{k}^{q}$ are the Jacobian matrix and measurement covariance matrix evaluated around $\boldsymbol{x}_{k \mid k-1} \cdot \boldsymbol{x}_{k \mid k-1}$ denotes the prediction state vector in the case of zero process noise [32]. Therefore, (36) is rewritten as

$$
\begin{aligned}
\boldsymbol{J}\left(\boldsymbol{x}_{k}^{q}\right)= & \left(\boldsymbol{Q}_{q}+\boldsymbol{F} \boldsymbol{J}^{-1}\left(\boldsymbol{x}_{k-1}^{q}\right) \boldsymbol{F}^{\mathrm{T}}\right)^{-1}+ \\
& \left(\widehat{\boldsymbol{H}}_{k}^{q}\right)^{\mathrm{T}}\left(\widehat{\boldsymbol{R}}_{k}^{q}\right)^{-1} \widehat{\boldsymbol{H}}_{k}^{q}
\end{aligned}
$$

\section{Dynamic antenna configuration}

The afore section shows that the target tracking performance is related to the antenna configuration in the collocated MIMO radar. For higher tracking precision, the dynamic antenna selection will be taken into account. The cognition idea can perceive the environment change by the closed loop feedback scheme from the transmitter to the receiver, and its superiority has been shown in many
MIMO radar resource management problems [11,12,2527]. Therefore, a closed loop antenna selection strategy is put forward in this section. The core of this strategy is to approximate the PCRLB by using the tracking information and then guide the antenna adjustment.

\subsection{Objective function}

To describe the sensor selection problem, we define the selection vector for transmit arrays and receive arrays at the $k$ th interval:

$$
\left\{\begin{array}{l}
\boldsymbol{t}_{s}^{k}=\left[t_{s 1}^{k}, t_{s 2}^{k}, \ldots, t_{s M}^{k}\right] \\
\boldsymbol{r}_{s}^{k}=\left[r_{s 1}^{k}, r_{s 2}^{k}, \ldots, r_{s N}^{k}\right]
\end{array}\right.
$$

where $t_{s m}^{k} \in\{0,1\}$ and $r_{s n}^{k} \in\{0,1\}$. Then, to describe the tracking performance of multiple targets, we choose the worst case of PCRLB as the cost function:

$$
\mathbb{F}\left(\boldsymbol{t}_{s}^{k}, \boldsymbol{r}_{s}^{k}\right)=\max _{q} \sqrt{\operatorname{tr}\left(\boldsymbol{J}^{-1}\left(\boldsymbol{x}_{k}^{q}, \boldsymbol{t}_{s}^{k}, \boldsymbol{r}_{s}^{k}\right)\right)}
$$

where $\operatorname{tr}(\cdot)$ denotes the trace operation. However, a problem in (39) is that the position, velocity and acceleration have heterogenous units, resulting in the trace operation possessing no meanings. Additionally, since the Doppler shift and DOA are our focus, the PCRLB in terms of velocity is extracted as the cost function. Though only the velocity estimate is used, the acceleration will feed into this by dynamics. Therefore, in the future, we will use (39) to represent the trace of PCRLB with respect to velocity.

There are many constraints in the antenna selection problem. First, to meet the anti-interception requirement, the active antennas are bounded:

$$
\sum_{m=1}^{M} t_{s m}^{k}+\sum_{n=1}^{N} r_{s n}^{k}=G
$$

where $G$ denotes the number of active antennas. Then, at least one transmit array and one receive array must be activated to keep the system being valid:

$$
\sum_{m=1}^{M} t_{s m}^{k} \geqslant 1, \quad \sum_{n=1}^{N} r_{s n}^{k} \geqslant 1
$$

As such, we formulate the optimization model as

$$
\begin{gathered}
\min \mathbb{F}\left(\boldsymbol{t}_{s}^{k}, \boldsymbol{r}_{s}^{k}\right) \\
\text { s.t. } \sum_{m=1}^{M} t_{s m}^{k}+\sum_{n=1}^{N} r_{s n}^{k}=G \\
\sum_{m=1}^{M} t_{s m}^{k} \geqslant 1, \quad \sum_{n=1}^{N} r_{s n}^{k} \geqslant 1 \\
t_{s m}^{k} \in\{0,1\}, \quad r_{s n}^{k} \in\{0,1\} .
\end{gathered}
$$


Considering the binary constraint in (42), the optimization model is known as NP-hard [28]. It can be solved via the examination of all combinations of transmitters and receivers. However, such computational burden is considerable. In what follows, an algorithm that integrates convex relaxation with local search is proposed as the solution strategy, which has lower computational complexity but comparable performance.

\subsection{Solution strategy integrated convex relaxation with local search}

We apply the convex relaxation to binary variables via linear programming and reformulate the optimization model as

$$
\begin{gathered}
\min \mathbb{F}\left(\boldsymbol{t}_{s}^{k}, \boldsymbol{r}_{s}^{k}\right) \\
\text { s.t. } \sum_{m=1}^{M} t_{s m}^{k}+\sum_{n=1}^{N} r_{s n}^{k}=G \\
\sum_{m=1}^{M} t_{s m}^{k} \geqslant 1, \quad \sum_{n=1}^{N} r_{s n}^{k} \geqslant 1 \\
0 \leqslant t_{s m}^{k} \leqslant 1, \quad 0 \leqslant r_{s n}^{k} \leqslant 1 .
\end{gathered}
$$

The relaxed optimization problem is convex, whose proof is given as follows.

Proof (43) is convex.

First, the constraints in (43) are linear and convex. Then, the objective function is

$$
\mathbb{F}\left(\boldsymbol{t}_{s}^{k}, \boldsymbol{r}_{s}^{k}\right)=\max _{q} \sqrt{\operatorname{tr}\left(\boldsymbol{J}^{-1}\left(\boldsymbol{x}_{k}^{q}, \boldsymbol{t}_{s}^{k}, \boldsymbol{r}_{s}^{k}\right)\right)} .
$$

Let $\boldsymbol{B}_{q}\left(\boldsymbol{t}_{s}^{k}, \boldsymbol{r}_{s}^{k}\right)=\sqrt{\operatorname{tr}\left(\boldsymbol{J}^{-1}\left(\boldsymbol{x}_{k}^{q}, \boldsymbol{t}_{s}^{k}, \boldsymbol{r}_{s}^{k}\right)\right)}$, the objective function is reformulated as

$$
\mathbb{F}\left(\boldsymbol{t}_{s}^{k}, \boldsymbol{r}_{s}^{k}\right)=\max _{q} \sqrt{\boldsymbol{B}_{q}\left(\boldsymbol{t}_{s}^{k}, \boldsymbol{r}_{s}^{k}\right)} .
$$

On the basis of (13) and the derivation of $\boldsymbol{R}_{k}^{q}$, we can rewrite $\boldsymbol{B}_{q}\left(\boldsymbol{t}_{s}^{k}, \boldsymbol{r}_{s}^{k}\right)$ as

$$
\begin{gathered}
\boldsymbol{B}_{q}\left(\boldsymbol{t}_{s}^{k}, \boldsymbol{r}_{s}^{k}\right)= \\
\operatorname{tr}\left[\left(\boldsymbol{J}_{P}+\sum_{m=1}^{M} \sum_{n=1}^{N} t_{s m} r_{s n} \boldsymbol{J}_{D}^{m, n}\left(\boldsymbol{x}_{k}^{q}\right)\right)^{-1}\right]
\end{gathered}
$$

where

$$
\boldsymbol{J}_{D}^{m, n}\left(\boldsymbol{x}_{k}^{q}\right)=\mathbb{E}_{\boldsymbol{x}_{k}^{q}}\left[\left(\boldsymbol{H}_{k}^{q}\right)^{\mathrm{T}} \operatorname{diag}\left\{\left(\boldsymbol{R}_{k}^{q}\right)_{11}^{-1},\left(\boldsymbol{R}_{k}^{q}\right)_{22}^{-1}\right\} \boldsymbol{H}_{k}^{q}\right]
$$

and $\operatorname{diag}\{\cdot\}$ denotes the $2 \times 2$ diagonal matrix.

In Swerling I model, we have

$$
\left(\boldsymbol{R}_{k}^{q}\right)_{11}^{-1}=\left(\boldsymbol{R}_{\mathrm{I} k}^{q}\right)_{11}^{-1}=\frac{32\left|\alpha_{k}^{q}\right|^{2} \pi^{2} T_{p}^{2}}{\sigma_{\boldsymbol{v}}^{2} \lambda^{2}} \frac{(L-1) L(2 L-1)}{6}
$$

$$
\begin{gathered}
\left(\boldsymbol{R}_{k}^{q}\right)_{22}^{-1}=\left(\boldsymbol{R}_{\mathrm{I} k}^{q}\right)_{22}^{-1}=\frac{2\left|\alpha_{k}\right|^{2} L}{\sigma_{\boldsymbol{v}}^{2}}\left(\frac{2 \pi}{\lambda}\right)^{2} . \\
\left(\left[\sin \theta_{k},-\cos \theta_{k}\right] \Delta \boldsymbol{b}_{m n}\left(\Delta \boldsymbol{b}_{m n}\right)^{\mathrm{T}}\left[\sin \theta_{k},-\cos \theta_{k}\right]^{\mathrm{T}}\right) .
\end{gathered}
$$

In Swerling II model,

$$
\begin{gathered}
\left(\boldsymbol{R}_{k}^{q}\right)_{11}^{-1}=\left(\boldsymbol{R}_{\mathrm{II} k}^{q}\right)_{11}^{-1}= \\
\frac{32 \pi^{2} T_{p}^{2}}{\sigma_{\boldsymbol{v}}^{2} \lambda^{2}}\left(\sum_{l=1}^{L}\left(\alpha_{k, l}^{q}\right)^{*} \alpha_{k, l}^{q}(l-1)^{2}\right) \\
\left(\boldsymbol{R}_{k}^{q}\right)_{22}^{-1}=\left(\boldsymbol{R}_{\mathrm{II} k}^{q}\right)_{22}^{-1}=\frac{2}{\sigma_{\boldsymbol{v}}^{2}}\left(\sum_{l=1}^{L} \alpha_{l}^{*} \alpha_{l}\right)\left(\frac{2 \pi}{\lambda}\right)^{2} . \\
\left(\left[\cos \theta_{k},-\sin \theta_{k}\right] \Delta \boldsymbol{b}_{m n}\left(\Delta \boldsymbol{b}_{m n}\right)^{\mathrm{T}}\left[\cos \theta_{k},-\sin \theta_{k}\right]^{\mathrm{T}}\right) .
\end{gathered}
$$

According to [33], we know that $\operatorname{tr}\left(\boldsymbol{X}^{-1}\right)$ is convex on the condition of $\boldsymbol{X} \in \boldsymbol{S}_{++}$, where $\boldsymbol{S}_{++}$is the set of symmetric positive definite matrices $[12,15]$. It is easy for us to prove that $\boldsymbol{J}_{P}+\sum_{m=1}^{M} \sum_{n=1}^{N} t_{s m} r_{s n} \boldsymbol{J}_{D}^{m, n}\left(\boldsymbol{x}_{k}^{q}\right)$ is a symmetric positive definite matrix on the condition that the initial $\boldsymbol{J}\left(\boldsymbol{x}_{1}^{q}\right) \in \boldsymbol{S}_{++}$. Thus, $\boldsymbol{B}_{q}\left(\boldsymbol{t}_{s}^{k}, \boldsymbol{r}_{s}^{k}\right)$ falls into the category of $\operatorname{tr}\left(\boldsymbol{X}^{-1}\right)$ with an affine transformation upon $t_{s m}$ and $r_{s n}$, and $\boldsymbol{B}_{q}\left(\boldsymbol{t}_{s}^{k}, \boldsymbol{r}_{s}^{k}\right)$ is convex. The corresponding pointwise maximum $\mathbb{F}\left(\boldsymbol{t}_{s}^{k}, \boldsymbol{r}_{s}^{k}\right)$ of $\boldsymbol{B}_{1}\left(\boldsymbol{t}_{s}^{k}, \boldsymbol{r}_{s}^{k}\right), \boldsymbol{B}_{2}\left(\boldsymbol{t}_{s}^{k}, \boldsymbol{r}_{s}^{k}\right), \ldots, \boldsymbol{B}_{q}\left(\boldsymbol{t}_{s}^{k}, \boldsymbol{r}_{s}^{k}\right)$ is also convex. Then, we know that the relaxed optimization problem in (43) is a convex problem, which completes the proof.

Therefore, (43) can be easily solved by standard convex optimization methods, e.g., interior point method [33]. It is noticeable that, the relaxed model does not equal the original one. That is because the solution of the relaxed problem may be fractional. However, owing to the feasible sets of the original model being contained in the feasible sets of the relaxed model, the optimal solution of the later one will not be bigger than the original one [29]. On the basis of these conditions, we then process the original problem as follows.

When the optimal sets of (43) are all integers, e.g., 0 and 1 , the optimal solutions are straightforward. When the optimal sets of (43) are fractional, we then apply a local search algorithm based on the optimal sets to gain higher precision.

The pseudo-code of the proposed algorithm is shown in Table 1. Firstly, we use the convex relaxation algorithm to generate high-quality initialized solutions. The mechanism of this algorithm is to select the most promising antennas as active ones which satisfy the constraints in (42). Then, 
we apply a local search to obtain solutions with higher precision. However, the efficiency of local search is mainly affected by the neighbor structure. Herein, we just tune one active antenna in the neighbor set from the current solution. The details can be depicted as follows.

Assume that the current active antenna set is $S_{\text {cur }}=$ $\left\{S_{\text {cur }}(1), S_{\text {cur }}(2), \ldots, S_{\text {cur }}(G)\right\}$, where $S_{\text {cur }}(g)$ indicates the position of each active antenna with $g=$ $1,2, \ldots, G$. Thus, all the neighbors of the current set is $S_{\text {nei }}^{i}=\left\{S_{\text {nei }}^{i}(1), S_{\text {nei }}^{i}(2), \ldots, S_{\text {nei }}^{i}(G)\right\}$, where $i=$ $1,2, \ldots,(M+N-G) \times G$. Since the solutions to the relaxed optimization problem in (43) indicate the promising degree of antennas to be activated, the local search will sequentially replace the antenna with the lowest order in $S_{\text {cur }}$ by the highest order in $S_{\text {nei }}^{i}$. This search scheme in one cycle will not stop until the objective function in (42) decreases. The local search will stop when all the active antennas in the initialized solution have been examined.

The following two remarks are appended in the application of the proposed method.

Remark 1 More antennas can be changed in the neighbor sets for better solution precision, however, it also leads to heavier computational burden. For example, when there are two antennas being changed, the number of neighbor sets is $\mathrm{C}_{M+N-G}^{2} \times \mathrm{C}_{G}^{2}$.

Remark 2 The sequential search in one cycle can stop via another criterion, i.e., the objective function in (42) no longer decreases. That means we must enumerate all the remaining antennas in one cycle of local search, which also results in more computational complexity.

The pseudo-code of the convex relaxation based method is as follows:

\section{Convex relaxation algorithm}

(i) Initialization. $\boldsymbol{t}_{0}^{k}=\mathbf{0}, \boldsymbol{r}_{0}^{k}=\mathbf{0}$

(ii) Acquire the solution $\boldsymbol{t}_{s}^{k}$ and $\boldsymbol{r}_{s}^{k}$ of the relaxed optimization problem (43) by convex optimization tools

(iii) Find $m=\operatorname{argmax}\left\{\boldsymbol{t}_{s}^{k}\right\}$

(iv) Update $\boldsymbol{t}_{s}^{k}(m)=0, \boldsymbol{t}_{0}^{k}(m)=1$

(v) Find $n=\arg \max \left\{\boldsymbol{r}_{s}^{k}\right\}$

(vi) Update $\boldsymbol{r}_{s}^{k}(n)=0, \boldsymbol{r}_{0}^{k}(n)=1$

(vii) Construct vectors $\boldsymbol{s}=\left[\boldsymbol{t}_{s}^{k}, \boldsymbol{r}_{s}^{k}\right]$ and $\boldsymbol{s}_{0}=\left[\boldsymbol{t}_{0}^{k}, \boldsymbol{r}_{0}^{k}\right]$

(viii) Sort $s$, choose the largest $G-2$ elements in $s$, set the corresponding positions in $s_{0}$ as 1 , and obtain the order index ind

(ix) Output the $\boldsymbol{t}_{0}^{k}$ and $\boldsymbol{r}_{0}^{k}$ as the initialized solutions for the local search algorithm

\section{Local search algorithm}

(i) Calculate the objective function $o b j_{\text {cur }}$ in terms of the initialized solutions, define the temporary objective function by using the temporary antenna set as $o b j_{\text {temp }}$, and set ite $=0$ (ii) for $i=1: G-2$

$$
\begin{aligned}
& \boldsymbol{s}_{0}(i n d(G-1-i))=0 \\
& \text { for } j=1: M+N-G \\
& \quad \boldsymbol{s}_{0}(i n d(G-2+j))=1 \\
& \quad \text { calculate } o b j_{\text {temp }} \text { by temporary antenna set } \boldsymbol{s}_{0} \\
& \text { if } o b j_{\text {temp }}<o b j_{\text {cur }} \\
& \quad o b j_{\text {cur }}=o b j_{\text {temp }} \text {, change active antenna sets }
\end{aligned}
$$

as $\boldsymbol{s}_{0}$

$$
\begin{aligned}
& \text { break } \\
& \text { end if } \\
& \text { end for } \\
& \text { end for }
\end{aligned}
$$

(iii) Apply (ii) to replace $\boldsymbol{s}_{0}(m)$ and $\boldsymbol{s}_{0}(n)$ respectively, and obtain better active antenna sets

\subsection{Computational complexity analysis}

The computational complexity of the proposed algorithm is estimated in this subsection. The above two algorithms in Section 4.2 shows that the relaxed problem plays a more important role in the computation cost, and the local search algorithm just serves as a supplement. When a standard convex optimization method is applied, e.g., interior point method [33], it requires at most $O(\sqrt{M N Q})$ iterations to achieve a given solution accuracy. The amount of work in each iteration is $O\left((M N Q)^{3}\right)$. By contrast, the exhaustive search [34] and heuristic search $[19,20]$ need $O\left(2^{M+N} Q\right)$ and $O(K M N(M+N) Q)$ iterations. Considering the matrix inverse operation in each iteration, whose computational complexity is $O\left((M N Q)^{3}\right)$ [35], the summary of computational complexity is given in Table 1 .

Table 1 Computational complexity comparison

\begin{tabular}{cc}
\hline Algorithm & Computational complexity \\
\hline Exhaustive search [34] & $O\left(2^{M+N}(M N)^{3} Q^{4}\right)$ \\
Heuristic search [20] & $O\left(K(M+N)(M N Q)^{4}\right)$ \\
Proposed algorithm & $O\left((M N Q)^{35}\right)$ \\
\hline
\end{tabular}

It can be seen that the proposed algorithm offers a fast solution strategy to adapt the system configuration to the environment. Especially in the collocated MIMO radar with large-scale arrays, significant computational savings are available.

\subsection{Closed loop feedback system for target tracking}

Due to the numerical stability and high precision [36,37], the SCKF is used for the nonlinear estimation. After acquiring the tracking result from the current time epoch, the PCRLB is predicted to guide the optimal antenna subset selection at next time epoch. The procedure is summarized as follows:

Step 1 Obtain the target state and the state estimate error covariance matrix of each target at current time epoch.

Step 2 Predict the PCRLB at next time epoch, and call the proposed strategy to adjust antenna selection. 
Step 3 The tracking results by the antenna adjustment are sent to guide the antenna configuration at next time epoch, rendering a closed loop feedback system.

\section{Simulations and results}

In this section, some target tracking results are given to illustrate the effectiveness of the proposed algorithm. All the simulations are run on a single Inter (R) Core (TM) i7-4790 CPU $(3.6 \mathrm{GHz})$ processor with $8 \mathrm{~GB}$ memory, Windows 7 OS. A collocated MIMO radar with uniformly distributed antennas with $1 \mathrm{~m}$ space in a $2 \mathrm{~m} \times 2 \mathrm{~m}$ region $(M=N=25)$, is chosen for analysis. Each MIMO radar array can both transmit and receive signals. The carrier wavelength is $\lambda=0.3 \mathrm{~m}$, and the number of antennas to be selected is $G=20$. A sequence of 30 frames with sample intervals $T_{s}=5 \mathrm{~s}$ is used in the entire simulation. There are $Q=3$ targets moving in the radar's surveillance region. The geometric relationship between the targets and the collocated MIMO radar is shown in Fig. 1.

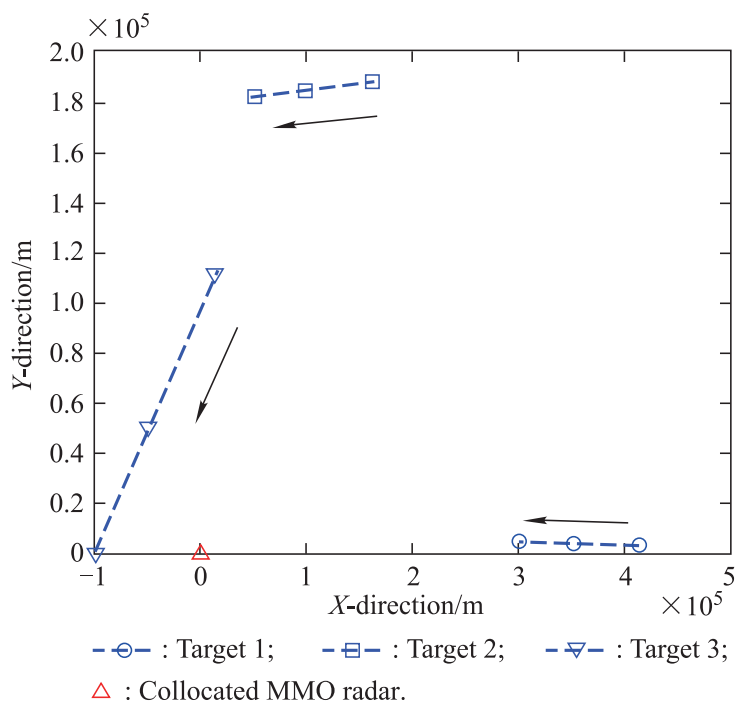

Fig. 1 Geometric relationship between targets and MIMO radar

The target initial states are shown in Table 2, and all the targets move in the CA model. The target reflectivity is $\alpha_{k, l}^{q}=1$, for $q=1,2,3, k=1,2, \ldots, 30$, in Swerling I model, and the mean reflectivity of targets is 1 in Swerling II model. The signal-to-noise ratio (SNR) is set as $-30 \mathrm{~dB}$ at the distance of $500 \mathrm{~km}$. The benchmark for comparison is the fixed antenna configuration (fixed configuration), where the first 10 antennas are selected for transmitting signals (within $x$ axis from $-2 \mathrm{~m}$ to $-1 \mathrm{~m}$ ) and the last 10 antennas are chosen for receiving signals (within $x$ axis from $1 \mathrm{~m}$ to $2 \mathrm{~m}$ ). The solutions yielded by the optimization model (42), which are carried out by the exhaustive search method [34] (exhaustive search) and heuristic search method (heuristic search) [20], are also presented for comparison. The criterions for performance are the PCRLB and the tracking root mean square error (RMSE) in terms of velocity:

$$
\mathrm{RMSE}_{k}=\max _{q} \frac{1}{N_{\text {sim }}} \sum_{j=1}^{N_{\text {sin }}} \sqrt{\left(\dot{x}_{k}^{q}-\widehat{\dot{x}}_{k}^{q}\right)^{2}+\left(\dot{y}_{k}^{q}-\widehat{\dot{y}}_{k}^{q}\right)^{2}}
$$

where $N_{\text {sim }}$ is the total number of Monte Carlo trials, $\left(\widehat{\dot{x}}_{k}^{q}, \widehat{\dot{y}}_{k}^{q}\right)$ denotes the velocity estimate for the $q$ th target in the $j$ th Monte Carlo trail [15].

Table 2 Target initial states

\begin{tabular}{cccc}
\hline Index & 1 & 2 & 3 \\
\hline Position $/ \mathrm{km}$ & $(412.975,2.95)$ & $(162.975,188.2)$ & $(12.68,111.79)$ \\
Velocity $/(\mathrm{m} / \mathrm{s})$ & $(-590,10)$ & $(-590,-40)$ & $(-591,-594)$ \\
Acceleration $/\left(\mathrm{m} / \mathrm{s}^{2}\right)$ & $(-2,0)$ & $(-2,0)$ & $(-2,-2)$ \\
\hline
\end{tabular}

\subsection{Swerling I model}

In this case, the target reflectivity follows with Swerling I model. To maintain the PCRLB of velocity and DOA on the same order of magnitude, we assume the number of pulses in once illumination is $L=300$ and pulse repetition period $T_{p}=0.01 \mathrm{~s}$. The representative selection results are shown in Fig. 2, and the comparison of performance, averaged by 100 Monte Carlo trails, is shown in Fig. 3.

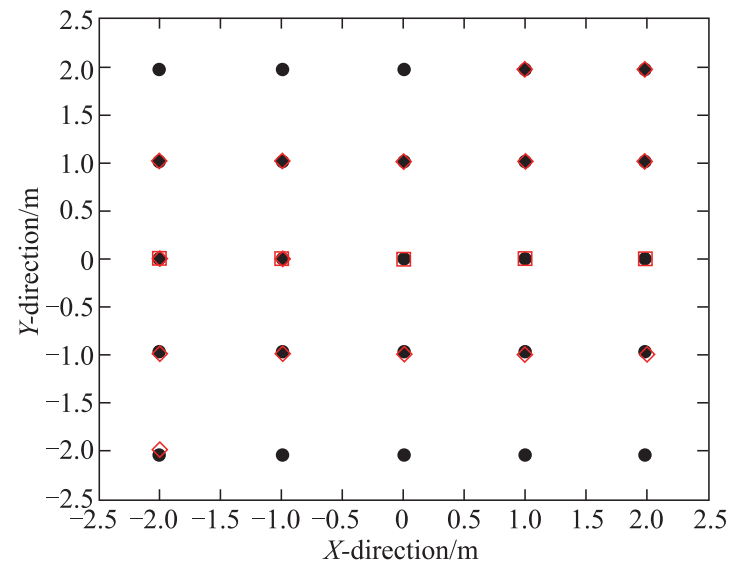

(a) Selection results at frame 5

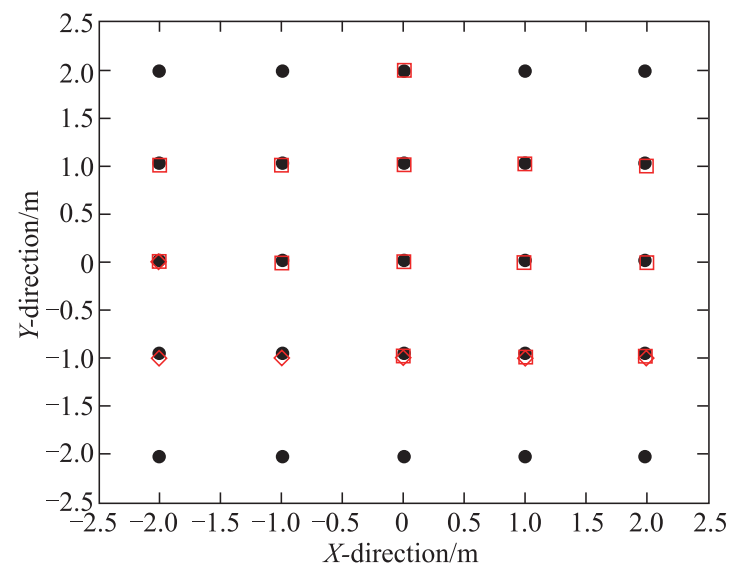

(b) Selection results at frame 15 


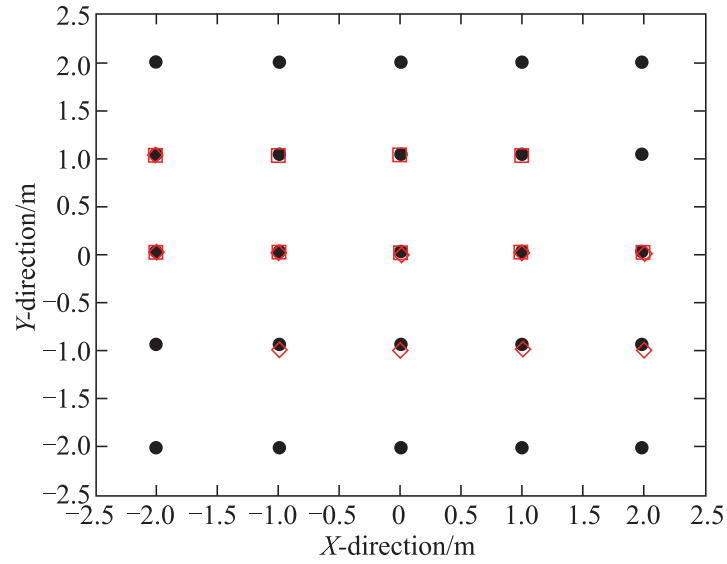

(c) Selection results at frame 25

- : MIMO array; $\square$ : Active transmitter; $\diamond$ : Active receiver.

Fig. 2 Representative array selection results for Swerling I model

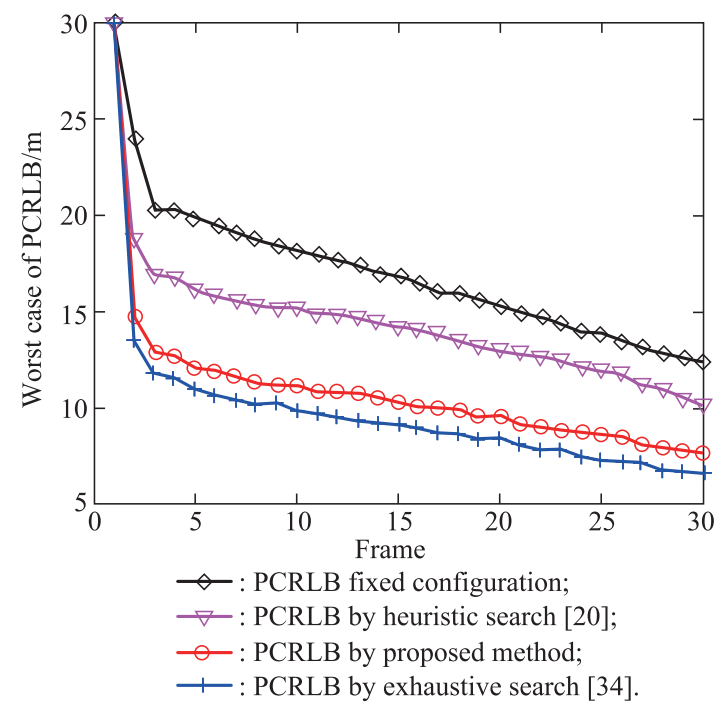

(a) PCRLB comparison

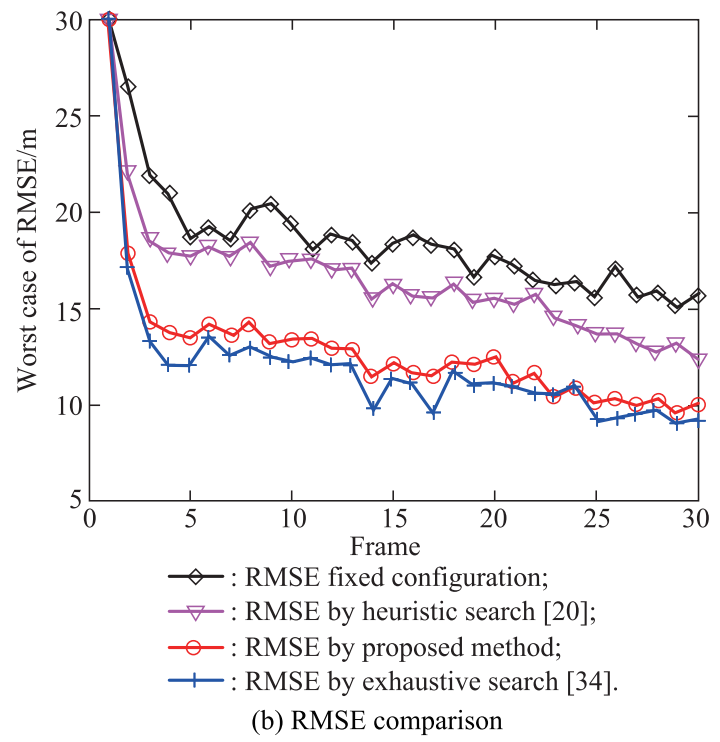

Fig. 3 Performance comparison in terms of the worst case of PCRLB and RMSE for Swerling I model
When the initial estimate error is set to be the same, the proposed strategy achieves much lower PCRLB and RMSE than the two other methods. Moreover, the close performance to the exhaustive search method is achieved. That is because the dynamic antenna selection provides the minimal measurement error according to the predictive PCRLB, which can result in the minimum worst case of PCRLB at each step. Hence, the tracking precision is obviously enhanced. By contrast, the fixed antenna configuration can only provide constant measurement error, and the target prior information is not fully employed. Thus, it offers higher PCRLB and RMSE. Moreover, the proposed strategy combines the convex relaxation technique with local search. The former technique supplies high quality of initialized solutions, and the local search is responsible for the higher solution precision. As such, better solutions are obtainable than the heuristic method.

In addition, the CPU time are compared among all the algorithms, as shown in Fig. 4. The results imply that the proposed strategy possesses much lower computational complexity than the heuristic method and exhaustive search method. Specifically, the proposed method takes about $5 \mathrm{~s}$ in the selection of optimal antenna sets in each frame, while it takes the heuristic search and exhaustive search about $10 \mathrm{~s}$ and $10^{3} \mathrm{~s}$, respectively. Such a result is consistent with the analysis in Section 4.3, where the proposed algorithm is very efficient.

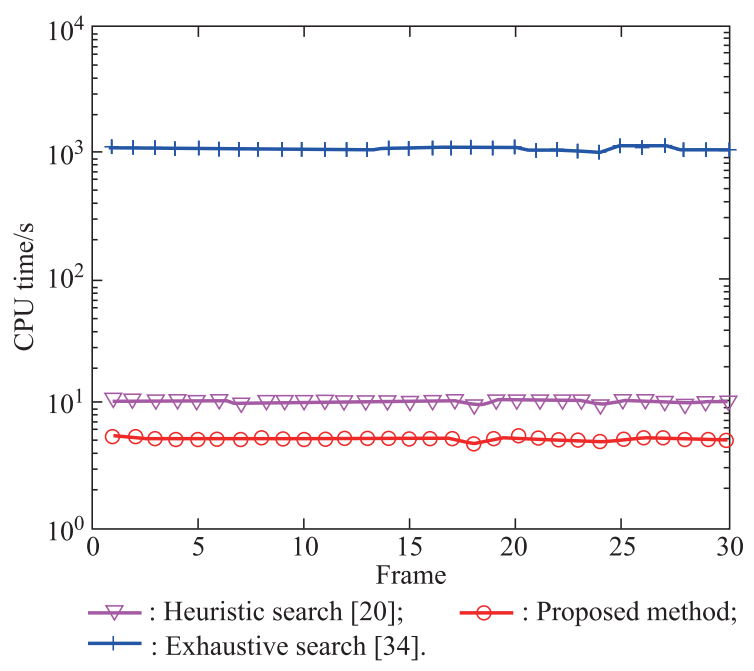

Fig. 4 CPU time comparison

Combining with Fig. 3, we can see that there is a balance between the performance and complexity among the three methods. However, the heavy computational load of the exhaustive search does restrict it to applications. That is because the number of collocated MIMO radar arrays is usually larger than 25 in practice, resulting in that the time consumption of exhaustive search cannot satisfy the real-time demand in the multi-target tracking. In this case, 
the proposed method is more preferable, which is due to its lower complexity and better performance.

To investigate the influence of SNR on the performance, Fig. 5(a) and Fig. 5(b) show the worst case of PCRLB and RMSE with different SNRs, respectively.

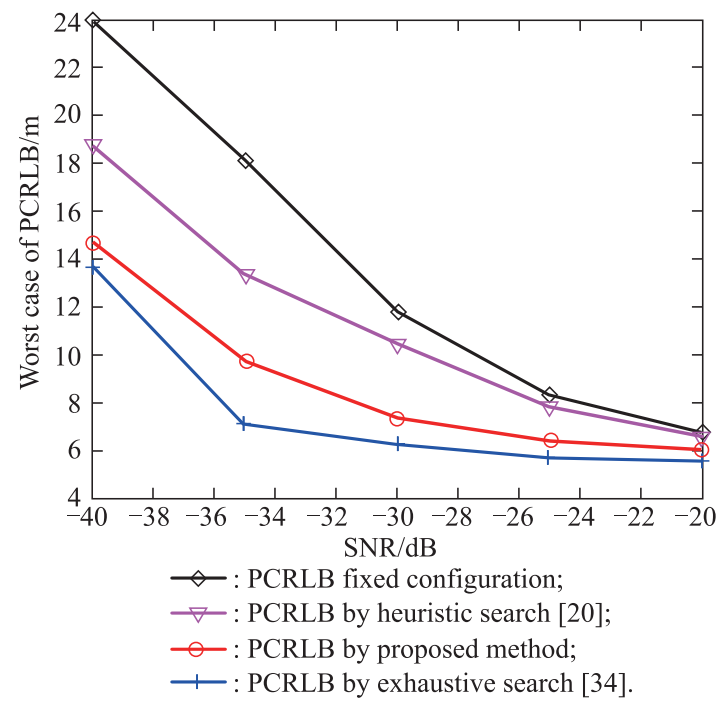

(a) PCRLB comparison

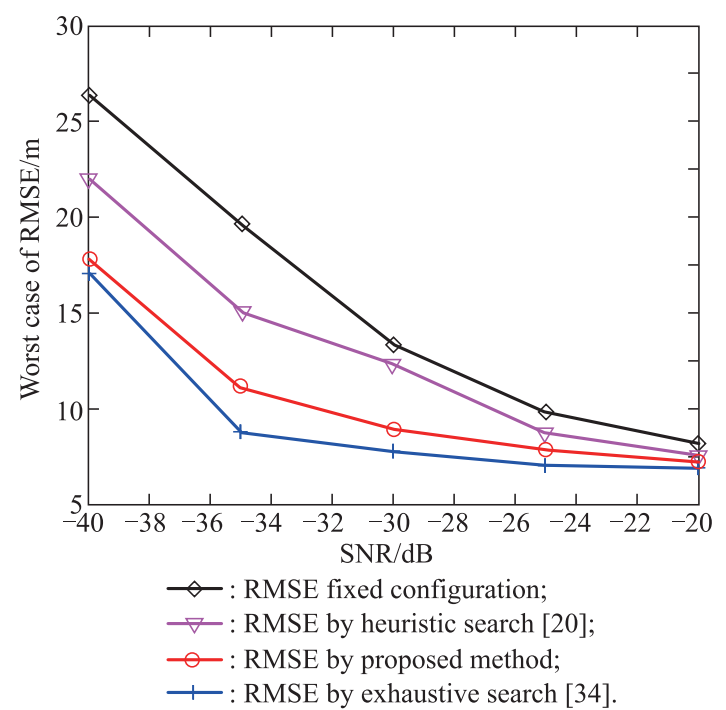

(b) RMSE comparison

Fig. 5 Performance comparison with the increase of SNR for Swerling I model

On the condition that SNR is increased, the performance of all the methods is improved. However, the proposed method still performs better than the fixed antenna configuration and the heuristic search method. Meanwhile, with the increase of SNR, the performance gap between the proposed method and the two methods is narrowed. In addition, the worst-case PCRLB and RMSE generated by the proposed method are very close to the exhaustive method, which demonstrates its robustness.

\subsection{Swerling II model}

When the target RCS follows with Swerling II model, the wide gap between the PCRLB of velocity and DOA should be emphasized. In order to maintain them on the same order of magnitude, we reset the simulation parameters as $L=100$ and $T_{p}=0.1 \mathrm{~s}$. The other parameters stay the same. The representative selection results are shown in Fig. 6, and the performance comparison is shown in Fig. 7.

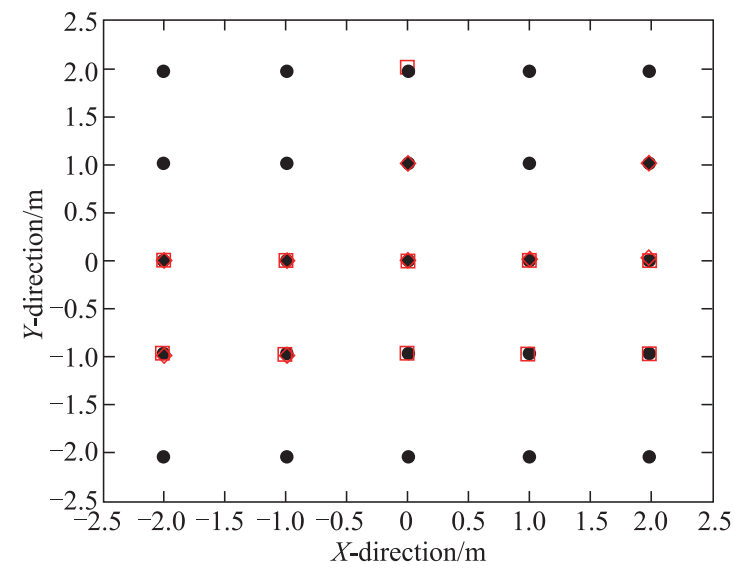

(a) Selection results at frame 5

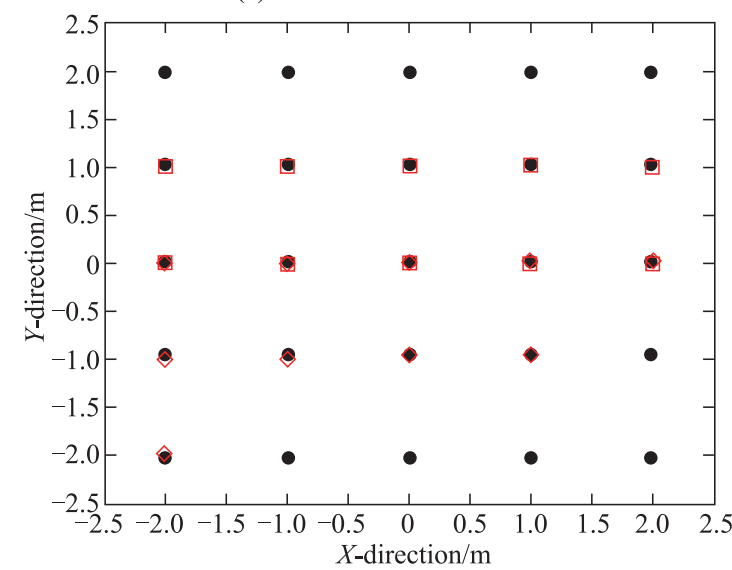

(b) Selection results at frame 15

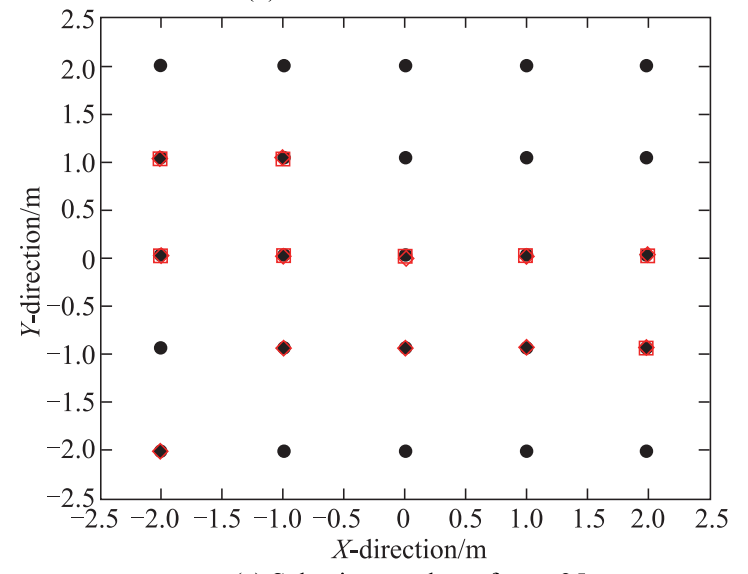

(c) Selection results at frame 25

- : MIMO array; $\quad \square$ : Active transmitter; $\diamond:$ Active receiver. Fig. 6 Representative array selection results for Swerling II model 


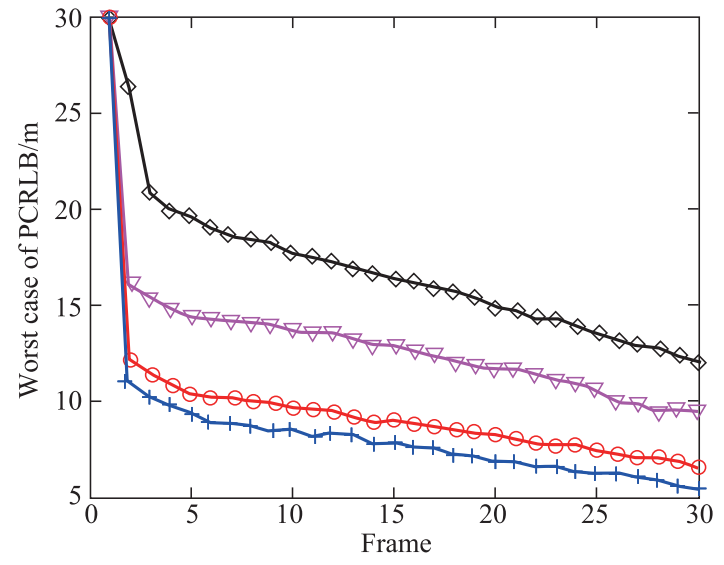

$\smile$ : PCRLB fixed configuration;

$\neg$ : PCRLB by heuristic search [20];

- : PCRLB by proposed method;

+ : PCRLB by exhaustive search [34].

(a) PCRLB comparison

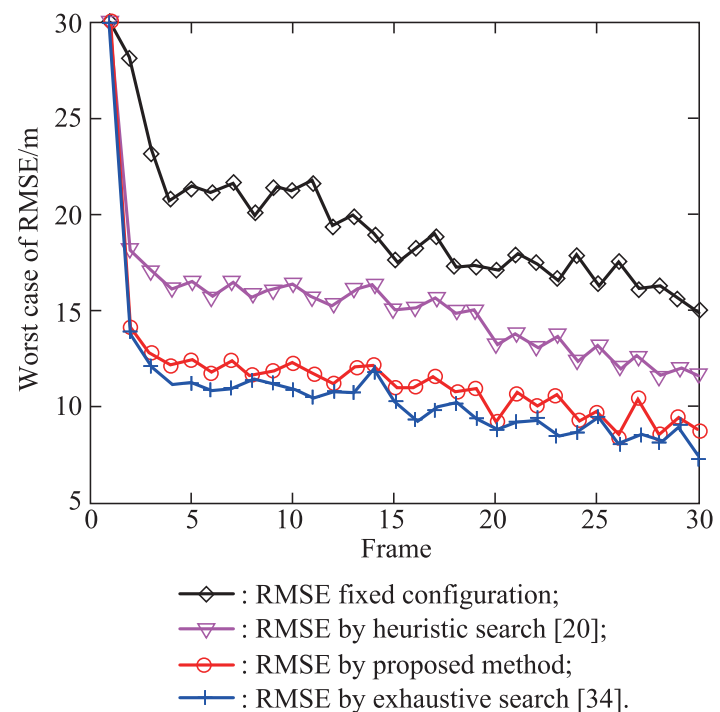

(b) RMSE comparison

Fig. 7 Performance comparison in terms of the worst case of PCRLB and RMSE for Swerling II model

It can be seen again that the proposed method achieves much lower RMSE and PCRLB than the fixed antenna configuration and the heuristic search method. That is because the proposed method can utilize the closed loop feedback scheme to adjust the antenna configuration, resulting in the minimum PCRLB being offered at each step. Moreover, the proposed method yields performance very similar to the exhaustive search method.

To reflect the influence of SNR on the performance, the results with the increase of SNR are presented in Fig. 8. Obviously, the PCRLBs and RMSEs of all the methods decrease along with the SNR being increased. However, the proposed method always provides a lower PCRLB and RMSE than the other two methods. On the other hand, the performance disparity between the exhaustive search method and the proposed method is very narrow, which verifies that the proposed method is very effective in the different SNR cases.

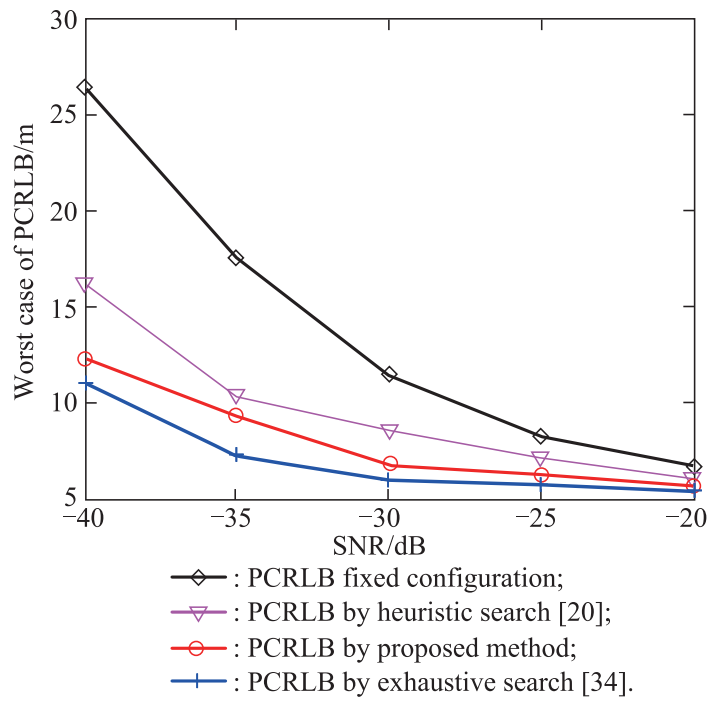

(a) PCRLB comparison

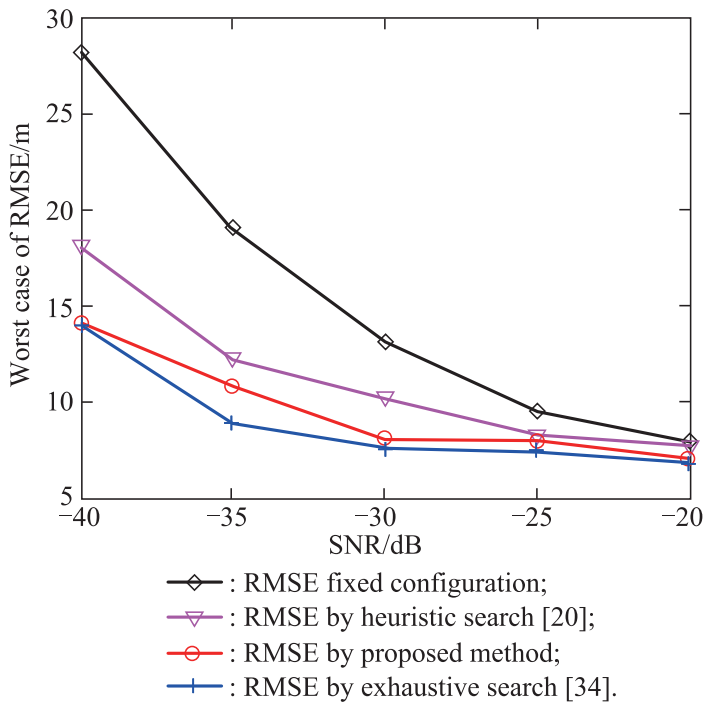

(b) RMSE comparison

Fig. 8 Performance comparison with the increase of SNR for Swerling II model

The following points may be deduced from the simulations.

(i) Whenever the target reflectivity being in Swerling I or Swerling II model, the tracking accuracy between the proposed method and the fixed antenna configuration narrows with the increase of SNR.

(ii) In comparison with the heuristic search and fixed antenna configuration, the proposed algorithm is able to achieve better and persistent performance.

(iii) Though the performance of the exhaustive search method is the best, the computational burden restricts 
its application in engineering. By contrast, the proposed method is efficient enough and can provide close-to optimal solutions.

\section{Conclusions}

An antenna adjustment scheme is developed in the multitarget tracking using collocated MIMO radar. The objective function is the derived PCRLB where the Doppler and DOA information are implied. In addition, two target RCS models are taken into account in the situation of the transmitting coherent pulse train. The formulated optimization model is known as NP-hard, and an efficient method is put forward for the solution to the problem. Simulation results depict the following points.

(i) The dynamic antenna selection strategy can offer much better performance than the fixed antenna selection and the heuristic search method.

(ii) The performance gap between the proposed method and the fixed antenna selection is narrowed with the increase of SNR.

(iii) The proposed method is efficient enough and can provide close-to optimal solutions, though a tradeoff between efficiency and optima is implied.

Future work includes the extension to the distributed MIMO radar and solving the problem via intelligence algorithms [38-40].

\section{References}

[1] FISHLER E, HAIMOVICH A M, BLUM R S, et al. Spatial diversity in radars-models and detection performance. IEEE Trans. on Signal Processing, 2006, 54(3): 823-838.

[2] STOICA P, LI J. MIMO radar with colocated antennas. IEEE Signal Processing Magazine, 2007, 24(5): 106-114.

[3] ZHANG H W, XIE J W, GE J A, et al. Optimization model and online task interleaving scheduling algorithm for MIMO radar. Computers \& Industrial Engineering, 2019, 127(1): 865-874.

[4] ZHANG H W, XIE J W, HU Q Y, et al. A hybrid DPSO with Levy flight for scheduling MIMO radar tasks. Applied Soft Computing, 2018, 71(11): 242-254.

[5] XU L Z, LI J, STOICA P. Target detection and parameter estimation for MIMO radar systems. IEEE Trans. on Aerospace Electronic Systems, 2008, 44(3): 927 - 939.

[6] YU Y, SUN S Q, MADAN R N, et al. Power allocation and waveform design for the compressive sensing based MIMO radar. IEEE Trans. on Aerospace Electronic Systems, 2014, 50(2): $898-909$.

[7] BEKKERMAN I, TABRIKIAN J. Target detection and localization using MIMO radars and sonars. IEEE Trans. on Signal Processing, 2006, 54(10): 3873-3883.

[8] LI J, XU L Z, STOICA P, et al. Range compression and waveform optimization for MIMO radar: a Cramer-Rao bound based study. IEEE Trans. on Signal Processing, 2008, 56(1): $218-232$.

[9] GORJI A A, KIRUBARAJAN T, THARMARSSA R. Optimal antenna allocation in MIMO radars with collocated antennas. IEEE Trans. on Aerospace Electronic Systems, 2014, 50(1):
$542-557$.

[10] GORJI A A, THARMARASA R, BLAIR W D, et al. Multiple unresolved target localization and tracking using collocated MIMO radars. IEEE Trans. on Aerospace Electronic Systems, 2012, 48(3): 2498-2517.

[11] YAN J K, JIU B, LIU H W, et al. Prior knowledge based simultaneous multibeam power allocation algorithm for cognitive multiple targets tracking in clutter. IEEE Trans. on Signal Processing, 2015, 63(2): 512-527.

[12] YAN J K, LIU H W, JIU B, et al. Simultaneous multibeam resource allocation scheme for multiple target tracking. IEEE Trans. on Signal Processing, 2015, 63(12): 3110-3122.

[13] JIN M, LIAO G S, LI J. Joint DOD and DOA estimation for bistatic MIMO radar. Signal Processing, 2009, 89(2): $244-$ 251.

[14] TICHAVSKY P, MURAVCHIK C, NEHORAI A. Posterior Cramer-Rao bounds for discrete-time nonlinear filtering. IEEE Trans. on Signal Processing, 1998, 46(5): 1386-1396.

[15] XIE M C, YI W, KIRUBARAJAN T. Receive-beam resource allocation for multiple target tracking with distributed MIMO radars. IEEE Trans. on Aerospace Electronic Systems, 2018, 54(5): $2421-2436$.

[16] BISHOP A N, FIDAN B, ANDERSON B D, et al. Optimality analysis of sensor-target localization geometries. Automatica, 2010, 46(3): 479-492.

[17] THARMARASA R, KIRUBARAJAN T, HERNANDEZ M L. Large-scale optimal sensor array management for multitarget tracking. IEEE Trans. on Systems, Man, and Cybernetics-Part C: Applications and Reviews, 2007, 37(5): 803-814.

[18] THARMARASA R, KIRUBARAJAN T, HERNANDEZ M L, et al. PCRLB-based multisensor array management for multitarget tracking. IEEE Trans. on Aerospace Electronic Systems, 2007, 43(2): 539-555.

[19] GODRICH H, PETROPULU A, POOR H V. A combinatorial optimization framework for subset selection in distributed multiple-radar architectures. Proc. of the IEEE International Conference on Acoustics, Speech, Signal Processing, 2011: 2796-2799.

[20] GODRICH H, PETROPULU A, POOR H V. Sensor selection in distributed multiple-radar architectures for localization: a knapsack problem formulation. IEEE Trans. on Signal Processing, 2012, 60(1): 247-260.

[21] HE Q, BLUM R S, GODRICH H, et al. Target velocity estimation and antenna placement for MIMO radar with widely separated antennas. IEEE Journal of Selected Topics in Signal Processing, 2010, 4(1): 79-100.

[22] SONG X Y, ZHENG N E, BAI T. Resource allocation schemes for multiple targets tracking in distributed MIMO radar systems. International Journal of Antennas and Propagation, 2017: 7241281.

[23] SONG X Y, ZHENG N E, YAN S H, et al. A joint resource allocation method for multiple targets tracking in distributed MIMO radar systems. EURASIP Journal on Advances in Signal Processing, 2018: 10.1155/2017/7241281.

[24] HAYKIN S, ZIA A, XUE Y, et al. Control theoretic approach to tracking radar: first step towards cognition. Digital Signal Processing, 2011, 21(6): 576-585.

[25] ZHANG H W, XIE J W, SHI J P, et al. Joint beam and waveform selection for the MIMO radar target tracking. Signal Processing, 2019, 156(3): $31-40$.

[26] CHAVALI P, NEHORAI A. Scheduling and power allocation 
in a cognitive radar network for multiple-target tracking. IEEE Trans. on Signal Processing, 2012, 60(2): 715-729.

[27] GARCIA N, HAIMOVICH A M, COULON M, et al. Resource allocation in MIMO radar with multiple targets for noncoherent localization. IEEE Trans. on Signal Processing, 2014, 62(10): $2656-2666$.

[28] MARTELLO S, TOTH P. Knapsack problems: algorithms and computer implementations. New York: Wiley, 1990.

[29] JOSHI S, BOYD S. Sensor selection via convex optimization. IEEE Trans. on Signal Processing, 2009, 57(2): 451-462.

[30] ARASARATNAM I, HAYKIN S, HURD T R. Cubature Kalman filtering for continuous-discrete systems: theory and simulations. IEEE Trans. on Signal Processing, 2010, 58(10): $4977-4993$.

[31] CHALLA S, MORELANDE M R, MUSICKI D, et al. Fundamentals of object tracking. Cambridge, UK: Cambridge University Press, 2011.

[32] GLASS J D, SMITH L D. MIMO radar resource allocation using posterior Cramer-Rao lower bounds. Proc. of the IEEE Aerospace Conference, 2011: 1-9.

[33] BOYD S, VANDENBERGHE L. Convex optimization. New York: Cambridge University Press, 2004.

[34] KELLERER H, PFERSCHY U, PISINGER D. Knapsack problems. Berlin: Springer, 2004.

[35] GOLUB G H, VAN LOAN C F. Matrix computations. 3rd ed. Baltimore: Johns Hopkins University Press, 1996.

[36] ZHANG H W, LIU W J, XIE J W, et al. Space-time allocation for transmit beams in collocated MIMO radar. Signal Processing, 2019, 164(11): $151-162$.

[37] ZHANG H W, XIE J W, SHI J P, et al. Sensor scheduling and resource allocation in distributed MIMO radar for joint target tracking and detection. IEEE Access, 2019, 7(1): $62387-$ 62400 .

[38] ZHANG H W, XIE J W, GE J A, et al. A hybrid adaptively genetic algorithm for task scheduling problem in the phased array radar. European Journal of Operational Research, 2019, 272(3): $868-878$.

[39] ZHANG H W, XIE J W, GE J A, et al. An entropy-based PSO for DAR task scheduling problem. Applied Soft Computing, 2018, 73(12): $862-873$.

[40] ZHANG H W, XIE J W, LU W L, et al. Novel ranking method for intuitionistic fuzzy values based on information fusion. Computers \& Industrial Engineering, 2019, 133(6): 139-152.

\section{Biographies}

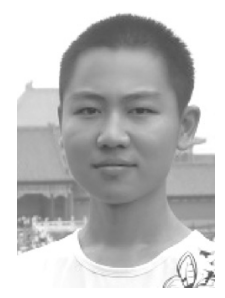

ZHANG Haowei was born in 1992. He received his bachelor and master degrees from the Air and Missile Defense College, Air Force Engineering University, Xi'an, Shaanxi, China, in 2014 and 2016, respectively. He is currently a doctoral student in the Air and Missile Defense College with the same university. His research interests include multifunction radar resource management and intelligent scheduling.

E-mail: zhw_xhzf@163.com

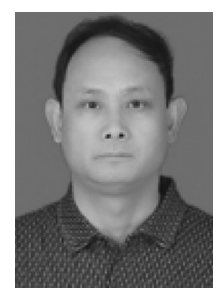

XIE Junwei was born in 1970. He received his bachelor, master, and doctor degrees from the Air and Missile Defense College, Air Force Engineering University, Xi' an, Shaanxi, China, in 1993, 1996 and 2009, respectively. He is currently a professor in the Air and Missile Defense College with the same university. He has published more than 100 refereed journal articles, book chapters, and conference papers. His research interests include novel radar systems as well as jamming and anti-jamming.

E-mail: xjw_xjw123@163.com

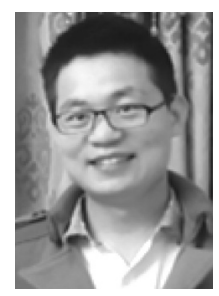

SHI Junpeng was born in 1991. He received his M.S. and doctor degrees from the Air Force Engineering University, Xi'an, Shaanxi, China, in 2014 and 2018 , respectively. He is currently a lecturer in the National University of Defense Technology, Anhui, Hefei. His research interests include array signal processing, compressive sensing, and radar antistealth technology.

E-mail: 15661081720@163.com

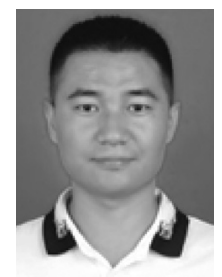

ZHANG Zhaojian was born in 1989. He received his bachelor, master and doctor degrees from the Air and Missile Defense College, Air Force Engineering University, Xi' an, Shaanxi, China, in 2011, 2013 and 2017, respectively. He is currently a lecturer in the Air Force Early Warning Academy of the PLA, Wuhan, Hubei. His research interest is radar signal processing. E-mail: zzj554038@163.com 